\title{
CINNAMOMUM GLANDULIFERUM Y C. VERUM (LAURACEAE) NATURALIZADAS EN LA ARGENTINA
}

\author{
GUSTAVO DELUCCHI ${ }^{1}$, HÉCTOR A. KELLER ${ }^{2}$ \& JULIO A. HURRELL ${ }^{3}$
}

\begin{abstract}
Summary: Delucchi, G., H. A. Keller \& J. A. Hurrell. 2016. Cinnamomum glanduliferum and C. verum (Lauraceae) naturalized in Argentina. Bonplandia 25(1): 33-41.

Two species of Cinnamomum Schaeff. (Lauraceae), C. glanduliferum (Wall.) Meisn. and C. verum J. Presl are mentioned for the first time as naturalized in Argentina. Species descriptions, distribution, phenology, vernacular names, uses, reference materials, and comments about their naturalization are included. Keys to differentiate the species of Cinnamomum in Argentina and the genera of Lauraceae naturalized in the country are also included. As a result of this contribution, a total of 19 species of Lauraceae, four of which are naturalized, are found in Argentina.
\end{abstract}

Key words: Argentine flora, Cinnamomum, Lauraceae, naturalization.

Resumen: Delucchi, G., H. A. Keller \& J. A. Hurrell. 2016. Cinnamomum glanduliferum y C. verum (Lauraceae) naturalizadas en la Argentina. Bonplandia 25(1): 33-41.

Esta contribución menciona por primera vez 2 especies de Cinnamomum Schaeff. (Lauraceae) naturalizadas en la Argentina: C. glanduliferum (Wall.) Meisn. y C. verum J. Presl. Se incluyen sus descripciones, distribución, fenología, nombres vernáculos, usos, materiales de referencia y comentarios sobre su naturalización. Asimismo, se incluyen claves para diferenciar las especies de Cinnamomum en la Argentina, y los géneros de Lauraceae naturalizadas en el país. Como resultado de esta contribución, el total de Lauraceae para la Argentina asciende a 19 especies, 4 de estas naturalizadas.

Palabras clave: Cinnamomum, flora Argentina, Lauraceae, naturalización.

\section{Introducción}

En este trabajo se cita por primera vez para la Argentina la presencia de dos especies naturalizadas del género Cinnamomum Schaeff. (Lauraceae): C. glanduliferum (Wall.) Meisn., "falso alcanforero", en la Ciudad Autónoma de
Buenos Aires y en la provincia de Buenos Aires, y C. verum J. Presl, "canelo", en la provincia de Misiones. El hallazgo de estas especies se realizó en el marco de distintos estudios florísticos y etnobotánicos vinculados con la naturalización de especies exóticas introducidas.

La familia Lauraceae Juss. incluye entre

${ }^{1}$ Facultad de Ciencias Naturales y Museo, Universidad Nacional de La Plata, Paseo del Bosque s.n., 1900-La Plata, Argentina. E-mail: delucchi@fcnym.unlp.edu.ar

${ }^{2}$ Facultad de Ciencias Forestales, Universidad Nacional de Misiones - Instituto de Botánica del Nordeste, Casilla de Correo 209, 3400-Corrientes, Argentina. E-mail: hakeller2000@yahoo.com.ar

${ }^{3}$ Laboratorio de Etnobotánica y Botánica Aplicada. Facultad de Ciencias Naturales y Museo, Universidad Nacional de La Plata, Calle 64 nro. 3, 1900-La Plata, Argentina. Investigador CONICET. E-mail: juliohurrell@gmail.com 
45-50 géneros y 2000-3500 especies, en su mayoría de las zonas cálidas del mundo, con unas pocas de zonas templadas (Rohwer, 1993). Para la Argentina se han citado siete géneros y 17 especies (Delucchi et al., 2007; Van der Werff et al., 2015; Poszkus Borrero et al., 2016), dos de las cuales son naturalizadas: Laurus nobilis L., en bosques de talas y ambientes modificados en las provincias de Buenos Aires y La Pampa (Williamson, 1967; Steibel et al., 2000; Delucchi et al., 2007), y Persea americana Mill., en ambientes alterados, capueras y zonas de desechos, en las provincias de Entre Ríos y Salta (Jozami \& Muñoz, 1982; Hadid de Cheda, 1991). Ambas especies también se hallaron en la Ciudad Autónoma de Buenos Aires, en la Reserva Costanera Sur: ejemplares 8-XI-2008, Hurrell et al. 6790 (LP), y 8-XII-2007, Hurrell et al. 6613 (LP), respectivamente.

\section{Materiales y Métodos}

Los resultados de esta contribución se basan en diversos viajes de estudio desde hace una década, que incluyeron relevamientos fotográficos y colecciones de materiales de referencia, depositados en los herbarios CTES (Instituto de Botánica del Nordeste, Corrientes) y LP (Plantas Vasculares, Museo de La Plata). Además, se consultaron distintos herbarios, bibliotecas y bases de datos digitales (www.theplantlist.org, www.tropicos.org, http://www.darwin.edu.ar).

En forma paralela, se realizaron relevamientos etnobotánicos basados en entrevistas abiertas y semiestructuradas a distintos pobladores locales, previo consentimiento informado (Albuquerque \& Lucena, 2004; Etkin \& Ticktin, 2010; Albuquerque et al., 2014), orientadas a obtener información acerca del cultivo y los usos locales de las especies tratadas, y datos sobre la dinámica de su naturalización (Hurrell \& Delucchi, 2013). Estos datos etnobotánicos complementaron los registros florísticos obtenidos. El mismo esquema ha sido aplicado en trabajos anteriores sobre especies naturalizadas en nuestro país (Hurrell et al., 2011, 2014; Keller et al., 2013; Stampella et al., 2014).

Para cada especie tratada se incluye su sinonimia, iconografía, descripción, distribución geográfica, fenología, polinización y dispersión, nombres vernáculos, usos, materiales de referencia y conclusiones sobre su naturalización. También se incluye una clave para diferenciar las especies de Cinnamomum en nuestro país, y una clave para distinguir los géneros de Lauraceae naturalizadas en la Argentina.

\section{Resultados}

Cinnamomum Schaeff., Bot. Exped.: 74. 1760; nom. cons.

Género con alrededor de 350 especies, la mayoría de las zonas tropicales, con mayor concentración en Asia, América Central y del Sur, y unas pocas especies de Australia y las islas del Pacífico (Rohwer, 1993; van der Werff, 1997; van der Werff \& Lorea, 1997; Li et al., 2008). En la Argentina, Cinnamomum se halla representado por una sola especie nativa de la provincia de Misiones, C. amoenum (Ness \& Mart.) Kosterm., localmente conocida como "canela", distribuida también en Brasil austral y en Uruguay (Van der Werff et al., 2015).

La siguiente clave permite distinguir las dos especies tratadas, $C$. glanduliferum y $C$. verum, y la especie nativa $C$. amoenum, a fin de diferenciar las tres especies del género que crecen en la Argentina.

\section{Clave para la identificación de las especies de Cinnamomum presentes en Argentina}

1. Hojas 3-nervias o pinnatinervias. Flores verdosas o amarillentas hasta de $3 \mathrm{~mm}$ long. Fruto globoso o elipsoide, cúpula ondulada.

2. Láminas foliares de 6-15 × 4-6,5 cm; pecíolos de 1,5-3,5 cm long. Inflorescencias de 4-10 cm long. Fruto globoso, cúpula ca. $10 \mathrm{~mm}$ diám. 
2'. Láminas foliares de 4-8 × 1-3 cm; pecíolos de 0,3-0,7 cm long. Inflorescencias de 2-4 cm long. Fruto elipsoide, cúpula de 2-4 $\mathrm{mm}$ long.

C. amoenum

1'. Hojas 3-5-nervias. Flores amarillas ca. 6 mm long. Fruto ovoide, cúpula dentada.

C. verum

1. Cinnamomum glanduliferum (Wall.) Meisn., Prodr. 15(1): 25. 1864. Fig. 1.

Laurus glandulifera Wall., Trans. Med. Soc. Calcutta 1: 45, 51, tab. 1. 1825; Camphora glandulifera (Wall.) Nees, P1. Asiat. Rar. 2: 72. 1831. 4-9.

Iconografia: Wu \& Hong, 2009: fig. 182,

Nombres vernáculos: "alcanforero", "falso alcanforero".

Árboles perennifolios, 5-15 (-20) m alt. Corteza castaño-grisácea, con olor alcanforado. Ramitas castaño-verdosas, robustas, las jóvenes anguladas, las mayores teretes. Yemas densamente seríceas. Hojas alternas; pecíolos 1,5-3,5 cm long., subglabros; láminas elípticas a ovado-elípticas, $6-15 \times 4-6,5 \mathrm{~cm}$, ápice agudo o cortamente acuminado, base cuneda o subredondeada, a veces asimétrica, coriáceas, cara superior más oscura y brillante, glabra, cara inferior glabra o pubescente, pinnatinervias $\mathrm{o}$ a veces subtrinervias, nervio medio y laterales conspicuos. Inflorescencias axilares paniculiformes, 4-10 $\mathrm{cm}$ long., pedúnculos y raquis glabros. Flores actinomorfas, bisexuales, hasta de $3 \mathrm{~mm}$ long., amarillentas. Perianto cupuliforme, tubo obcónico, lobos 6, ovados, subiguales. Estambres 9 ( 3 ciclos), ca. 1,5 mm long., filamentos pubescentes, los del tercer ciclo con 2 glándulas basales. Ovario ca. 1,2 $\mathrm{mm}$ long., glabro. Fruto globoso, hasta de $1 \mathrm{~cm}$ diám., negro, cúpula angostamente obcónica, ondulada.

Distribución geográfica: Especie de la India, Bután, Nepal, China continental, Birmania y Malasia; crece principalmente en bosques perennifolios latifoliados, entre $1500-3000 \mathrm{~m}$ s. n. m. (Li et al., 2008). Se ha señalado como naturalizada en Europa (Randall, 2012). En la
Argentina, fue hallada en la Ciudad Autónoma de Buenos Aires y en localidades de la provincia de Buenos Aires.

Fenología, polinización y dispersión: Florece en primavera y fructifica en verano. La polinización es entomófila; la diseminación, ornitocora.

Usos: Ornamental, para parques y plazas; soporta las heladas, se reproduce por semillas y su crecimiento es rápido (Dimitri, 1987; Valla et al., 2005). La madera se utiliza para la construcción de muebles. Raíces y corteza se emplean en medicina popular, en linimentos, como rubefaciente y antiséptico. Presenta actividad antimicrobiana (Singh et al., 2013). Las ramitas contienen aceite esencial y alcanfor. Es una fuente secundaria de este último, que se obtiene principalmente de Cinnamomum camphora (L.) J. Presl (Del Vitto et al. 1998; Li et al., 2008).

Material examinado: ARGENTINA. Buenos Aires: Pdo. Berazategui, Parque Pereyra Iraola, 20X-1998, Delucchi 1863 (LP); Pdo. Gral. Belgrano, Estancia Santa Narcisa, 26-VIII-2004, Delucchi 2917 (LP); Pdo. Monte, Monte, quinta, 15-XII-2015, Hurrell \& Bazzano 7038 (LP). Capital Federal: Reserva Costanera Sur, 23-XI-2007, Delucchi 3185 (LP); ibíd., 28-XII-2007, Delucchi 3203 (LP); ibíd., 8-XII-2007, Hurrell et al. 6610 (LP).

2. Cinnamomum verum J. Presl, Přir. Rostlin 2(2): 36. 1825. Fig. 2.

Laurus cinnamomum L., Sp. Pl. 1: 369. 1753; Cinnamomum zeylanicum Blume, Bijdr. Fl. Ned. Ind. 11: 568. 1825. Camphora mauritiana Lukman., Nomencl. Icon. Cann. 22. 1889. 


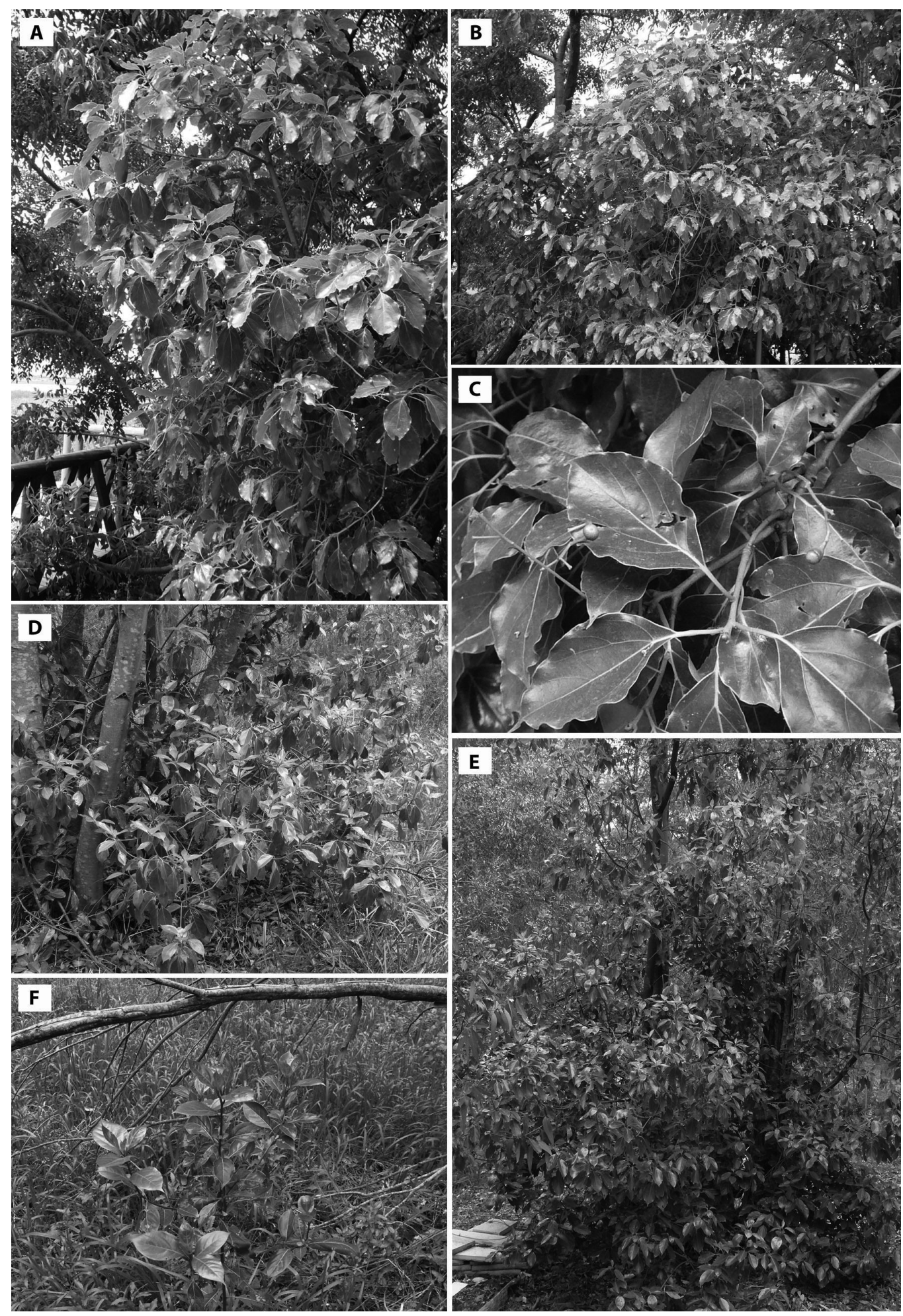

Fig. 1. Cinnamomum glanduliferum. A-B: Árboles en la Reserva Costanera Sur, Ciudad Autónoma de Buenos Aires. C: Hojas y frutos inmaduros. D-F: Ejemplares y renovales en San Miguel del Monte, provincia de Buenos Aires (Fotos: D. H. Bazzano). 
Iconografía: Vietz, 1800: tab. 61; Sims, 1819: tab. 2028; Köhler, 1887: tab. 78.

Nombres vernáculos: "árbol de la canela", "canelero", "canelo", "canelo de Ceilán”.

Árboles perennifolios, hasta $10 \mathrm{~m}$ alt. Corteza castaño-negruzca, con sabor a aldehído cinámico en el interior. Ramitas grisáceas, albo-maculadas, tetrágonas. Yemas seríceo-puberulentas. Hojas opuestas, a veces alternas; pecíolos ca. 2 cm long., glabros; láminas ovadas u ovado-elípticas, 11-16 × 4,5-5,5 cm, ápice acuminado, base cuneda, coriáceas o subcoriáceas, glabras en ambas caras, la superior verde brillante, la inferior verdoso-blancuzcas, 3-5-nervias, nervio medio y laterales muy marcados. Inflorescencias terminales o axilares paniculiformes, 10$12 \mathrm{~cm}$ long., pedúnculos y raquis seríceopuberulentos. Flores actinomorfas, bisexuales, ca. $6 \mathrm{~mm}$ long., amarillas. Perianto cupuliforme, tubo obcónico, lobos 6, oblongos, subiguales. Estambres 9 (3 ciclos), ca. $1 \mathrm{~mm}$ long., filamentos pilosos, los del tercer ciclo con 2 glándulas basales. Ovario 10-15 mm long., glabro. Fruto ovoide, 1-1,5 cm diám., negro, cúpula dilatada, dentada.

Distribución geográfica: Especie nativa de Sri Lanka, de amplia difusión en cultivo en diversas zonas cálidas del mundo ( $\mathrm{Li}$ et al., 2008). Se ha naturalizado en los Estados Unidos, Australia e islas del Pacífico (Randall, 2012). En la Argentina, se encuentra naturalizada en Misiones.

Fenología, polinización y dispersión: Florece en primavera y principios del verano, fructifica en verano. La polinización es entomófila; la diseminación, ornitocora.

Usos: La corteza, las ramitas hojosas y los frutos inmaduros contienen aceite esencial. La corteza seca es fuente de la importante especia llamada "canela", también empleada en medicina popular desde el antiguo Egipto, como remedio estimulante, estomáquico, carminativo, antiinflamatorio bronquial, antitusivo, antiséptico (Hurrell et al., 2008; Li et al., 2008). Presenta, entre otros, efectos antidiabético (Ponnusamy et al., 2011), antioxidante y analgésico (Pandey \& Chandra, 2015), anti-Trypanosoma cruzi (Azeredo et al., 2014), antibacteriano (Yap et al., 2015), antimicótico (Varadarajan et al., 2015), antihelmíntico (Williams et al., 2015).

Material examinado: ARGENTINA. Misiones: Dep. Candelaria, Loreto, $27^{\circ} 22^{\prime} 0,24^{\prime \prime} \mathrm{S}-55^{\circ}$ 31' 25,27' W, 5-VIII-2014, Keller \& Franco 12138 (CTES). Dep. Eldorado, Eldorado, Parque Schwelm, 10-IX-2013, Keller \& Rivero 11582 (CTES); Km 3, alrededores del autódromo, 12-IX2013, Keller \& Pirelli 11583 (CTES).

\section{Discusión y Conclusiones}

La naturalización de especies exóticas es un tema central en diversos estudios en todo el mundo. La tendencia actual de estos estudios se basa en considerar la naturalización como un proceso, que puede derivar o no en una invasión (Richardson et al., 2011). En este sentido, el primer registro de especies naturalizadas es relevante para evaluar su potencial invasor en el futuro. Las plantas naturalizadas son exóticas capaces de mantener poblaciones por varios ciclos de vida sin intervención humana directa, al menos por 10 años, mediante sus propios mecanismos de propagación, sea por vía reproductiva o vegetativa (Richardson et al., 2000, 2010, 2011; Pyšek et al., 2004; Pyšek \& Richardson, 2006). Según este marco, las observaciones realizadas en el terreno y los testimonios de los informantes locales entrevistados, las dos especies de Cinnamomum tratadas se encuentran naturalizadas en la Argentina.

Cinnamomum glanduliferum ha sido hallada en fisonomías boscosas secundarias de la Reserva Costanera Sur, Ciudad Autónoma de Buenos Aires, donde es frecuente, y en la provincia de Buenos Aires, en los bosques del Parque Pereyra Iraola, Berazategui, donde es abundante en el sotobosque, en una estancia de General Belgrano, y en Monte, donde se encuentran renovales y adultos en bosques implantados de eucaliptos, posteriormente abandonados. Seguramente su presencia se debe a las aves que dispersan sus semillas. 

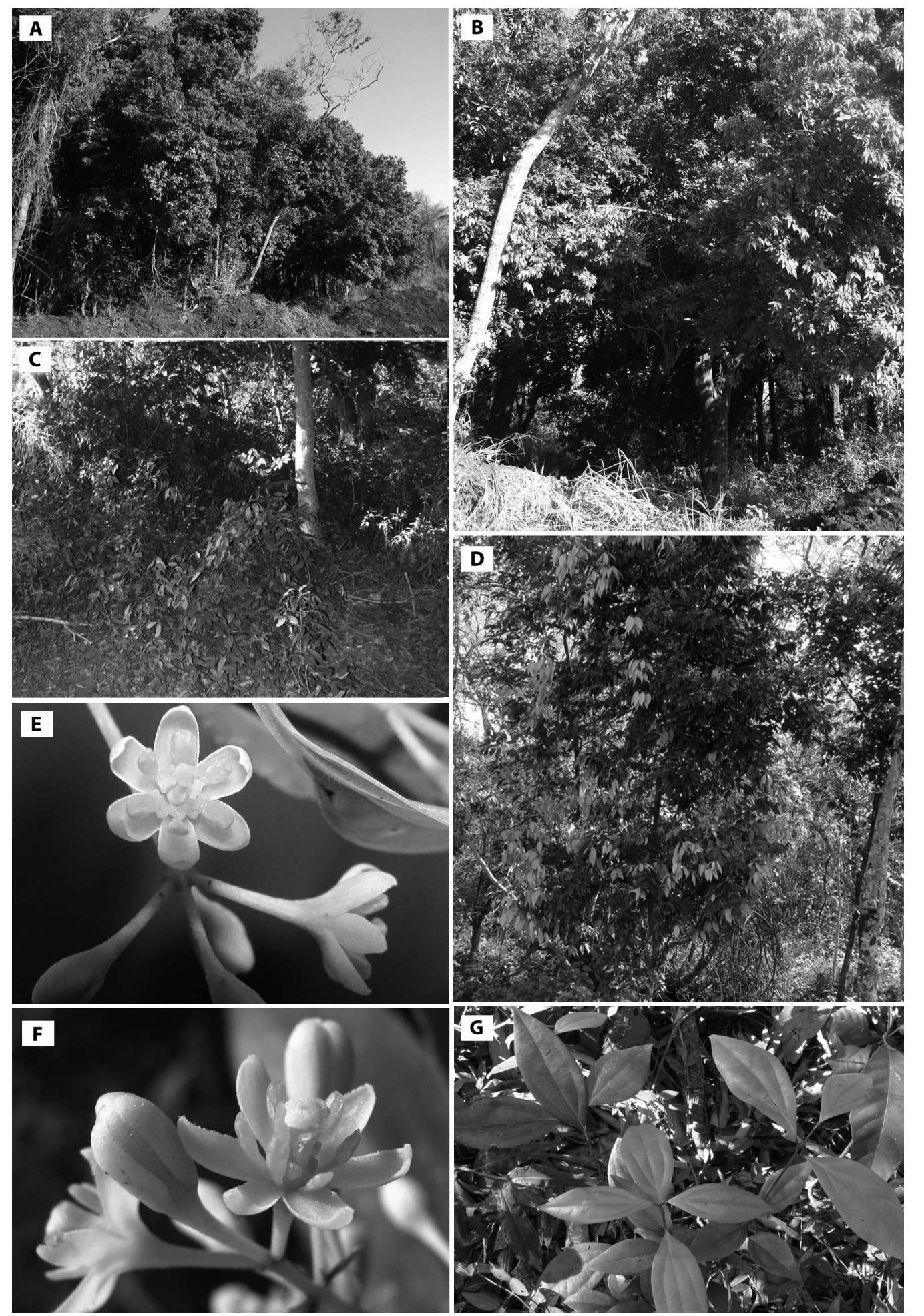

Fig. 2. Cinnamomum verum en Eldorado, Misiones. A-B: Aspectos de árboles adultos. C-D: Aspecto de renovales de distintos tamaños. E-F: Detalles de las flores. G: Hojas 3-nervias de plántulas producto de la regeneración espontánea (Fotos: H. A. Keller). 
Cinnamomum verum ha sido encontrada en la provincia de Misiones, principalmente en capueras y bosques secundarios en el distrito de las selvas. En esos sectores tienen abundante regeneración espontánea a partir de semillas; asimismo, se observan árboles que generalmente rebrotan de cepa, lo que dificulta su remoción. En el sur de Misiones crecen individuos de esta especie en mogotes de selva rodeados de pastizales, seguramente antropogénicos (taperas antiguas). En esta provincia los cultivos de "canelos" fueron mencionados por Dimitri(1987), quien también indicó que se reproducen bien por semillas. No obstante, individuos pertenecientes a esta especie se cultivan a menudo en patios y jardines con fines ornamentales y para sombra, y no para utilizarla con fines condimenticios: por lo general, los pobladores locales entrevistados no identifican estos árboles con la "canela", especia que adquieren en mercados.

Según los resultados, son cuatro las especies naturalizadas de Lauraceae en nuestro país: Cinnamomum glanduliferum, $C$. verum, Laurus nobilis y Persea americana. Asimismo, el total de Lauraceae para la Argentina es de 19 especies. La siguiente clave permite diferenciar los tres géneros con especies naturalizadas.

\section{Clave para diferenciar los géneros de Lauraceae con especies naturalizadas en Argentina}

1. Flores unisexuales, 2-meras, perianto 4-lobado. Inflorescencias umbeliformes.

Laurus

1'. Flores bisexuales, 3-meras, perianto 6-lobado. Inflorescencias paniculiformes.

2. Lobos del perianto desiguales (los 3 externos más cortos). Frutos ovoides a piriformes de 8-18 cm long.

Persea

2'. Lobos del perianto iguales. Frutos globosos u ovoides de 1-1,5 cm long.

Cinnamomum

\section{Agradecimientos}

Al personal de los herbarios CTES y LP, y de la biblioteca y herbario SI (Instituto de Botánica Darwinion, San Isidro); a Daniel H. Bazzano por facilitarnos material fotográfico, a los revisores anónimos por sus oportunas sugerencias, y a los informantes entrevistados que, de forma desinteresada, aportaron valiosa información para la resolución de este trabajo.

\section{Bibliografía}

ALBUQUERQUE, U. P. \& R. F. LUCENA. 2004. Métodos e técnicas na pesquisa etnobotânica. 190 pp. Livro Rápido/NUPEEA, Recife.

ALBUQUERQUE, U. P., L. V. F. CRUZ DA CUNHA, R. F. P. LUCENA \& R. F. P. ALVES (eds.) 2014. Methods and techniques in Ethnobiology and Ethnoecology. Springer-Humana Press, New York.

AZEREDO, C. M., T. G. SANTOS, B. H. MAIA \& M. J. SOARES. 2014. In vitro biological evaluation of eight different essential oils against Trypanosoma cruzi, with emphasis on Cinnamomum verum essential oil. BMC Complement. Altern. Med. 14: 309, doi: 10.1186/1472-6882-14-309.

DEL ViTTO, L. A., E. M. PETENATTI \& M. E. PETENATTI. 1998. Recursos herbolarios de San Luis (Argentina). Segunda Parte. Plantas exóticas cultivadas, adventicias y/o naturalizadas. Multequina 7: 29-48.

DELUCCHI, G., E. FARINA \& S. TORRES ROBLES. 2007. Laurus nobilis (Lauraceae) especie naturalizada en la República Argentina. Bol. Soc. Argent. Bot. 42: 309-312.

DIMITRI, M. J. 1987. Lauráceas. Encicl. Argent. Agric. Jard. I (1): 395-399. Acme, Buenos Aires.

ETKIN, N. L. \& T. TICKTIN. 2010. Advancing an ethnoecological perspective that integrates theory and method in ethnobotany. En U. P. Albuquerque \& N. Hanazaki (eds). Recent developments and case studies in Ethnobotany, pp. 33-57. SBEE-NUPEEA. Recife.

HADID DE CHEDA, M. 1991. Lauraceae. En L. J. Novara (ed.). Flora del Valle de Lerma. Aportes Botánicos de Salta. Ser. Flora 1: 1-7.

HURRELL, J. A. \& G. DELUCCHI. 2013. Aportes de la Etnobotánica al estudio de las invasiones biológicas. Casos en la región rioplatense (Argentina). Revista Historia Natural (3ra. serie) 3: 61-76.

HURRELL, J. A., E. A. ULIBARRI, G. DELUCCHI \& M. L. POCHETTINO. 2008. Plantas aromáticas condimenticias. En J. A. Hurrell (ed.), Biota 
Rioplatense XIII. 268 pp. Editorial LOLA, Buenos Aires.

HURRELL, J. A., G. DELUCCHI \& H. A. KELLER. 2011. Carya illinoinensis (Juglandaceae), adventicia en la Argentina. Bonplandia 20: 47-54.

HURRELl, J. A., CABANILlAS, P., GUERRERO, E. \& G. DELUCCHI. 2014. Naturalización y etnobotánica de Vitis labrusca L. (Vitaceae) en la región rioplatense, Argentina. Rev. Mus. Argentino Cienc. Nat., n.s. 16: 13-18.

JOZAMI, J. M. \& J. D. MUÑOZ. 1982. Árboles y arbustos indígenas de la Prov. de Entre Ríos. 407 pp. IPNAYS CONICET-UNL, Santa Fe.

KELLER, H. A., P. C. STAMPELLA, G. DELUCCHI \& J. A. HURRELL. 2013. Vernicia fordii y Aleurites moluccanus (Euphorbiaceae) en la Argentina. Naturalización y Etnobotánica. Bol. Soc. Argent. Bot.: 553-561.

KÖHLER, F. E. 1887. Medizinal Pflanzen 1: tab. 78. Köhler, Gera-Untermhaus.

LI X., J. LI \& H. VAN DER WERFF. 2008. Cinnamomum. En Z. Y. Wu, P. H. Raven \& D. Y. Hong (eds.), Flora of China 7: 102-254. Science Press, Beijing-Missouri Botanical Garden Press, St. Louis.

PANDEY, M. \& D. R. CHANDRA. 2015. Evaluation of ethanol and aqueous extracts of Cinnamomum verum leaf galls for potential antioxidant and analgesic activity. Indian J. Pharm. Sci. 77: 243247.

PONNUSAMY, S., R. RAVINDRAN, S. ZINJARDE, S. BHARGAVA \& A. RAVI KUMAR. 2011. Evaluation of traditional Indian antidiabetic medicinal plants for human pancreatic amylase inhibitory effect in vitro. Evid. Based Complement. Alternat. Med. Doi: 10.1155/2011/515647.

POSZKUS BORRERO, P. A., A. V. BOHREN, H. A. KELLER, L. A. GRANCE \& C. J. DUMMEL. 2016. La arquitectura foliar de las especies de Lauraceae Juss. nativas de Misiones, Argentina. Bol. Soc. Argent. Bot. 51: 37-57.

PYŠEK, P. \& D. RICHARDSON. 2006. The biogeography of naturalization in alien plants. J. Biogeography 12: 2040-2050.

PYŠEK, P., D. RICHARDSON, M. REJMÁNEK, G. WEBSTER, M. WILLIAMSON \& J. KIRSCHNER. 2004. Alien plants in checklists and floras: towards better communication between taxonomists and ecologists. Taxon 53: 131-143.

RANDALL, R. P. 2012. A Global Compendium of Weeds. 2nd ed. Department of Agriculture and Food, Western Australia, Perth.

RICHARDSON, D. M., P. PYŠEK, M. REJMÁNEK, M. G. BARBOUR, F. DANE PANETTA \& C. J. WEST. 2000. Naturalization and invasion of alien plants: concepts and definitions. Diversity Distrib. 6: 93-107.
RICHARDSON, D. M., C. C. DAEHLER, M. R. LEISHMAN, A. PAUCHARD \& P. PYŠEK. 2010. Plant invasions: theoretical and practical challenges. Biol. Invasions 12: 3907-3911.

RICHARDSON, D. M., P. PYŠEK \& J. T. CARLTON. 2011. A compendium of essential concepts and terminology in invasion ecology. En R. M. Richardson, (ed.), Fifty Years of Invasion Ecology: The Legacy of Charles Elton, pp. 409420. Wiley-Blackwell, Oxford.

ROHWER, J. G. 1993. Lauraceae. En K. Kubitzki (ed.). The families and genera of vascular plants. II: 366391. Springer, New York-Berlin.

SIMS, J. 1819. Curtis's Botanical Magazine 46: tab. 2028. Couchman, London.

SINGH, C., S. SINGH, C. PANDE, G. TEWARI, V. PANDE \& P. SHARMA. 2013. Exploration of antimicrobial potential of essential oils of Cinnamomum glanduliferum, Feronia elephantum, Bupleurum hamiltonii and Cyclospermum leptophyllum against foodborne pathogens. Pharm. Biol. 51: 1607-1610.

STAMPELlA, P. C., G. DELUCCHI, H. A. KELLER \& J. A. HURRELL. 2014. Etnobotánica de Citrus reticulata (Rutaceae, Aurantioideae) naturalizada en la Argentina. Bonplandia 23: 151- 162.

STEIBEL, P. E., H. O. TROIANI \& T. WILLIAMSON. 2000. Agregados al catálogo de las plantas naturalizadas y adventicias de la provincia de La Pampa, Argentina. Rev. Fac. Agron. UNLPam 11: 75-90.

VALLA, J. J., L. JANKOWSKI, D. BAZZANO \& A. J. HERNÁNDEZ. 2005. Árboles urbanos. En J. A. Hurrell (ed.), Biota Rioplatense IV. 1ra. Reimpr., 320 pp. Editorial LOLA, Buenos Aires.

VAN DER WERFF, H. 1997. Lauraceae. En Fl. of North America Edit. Committee (eds.), F1. of North America North of Mexico 3: 26-36. Oxford University Press, New York.

VAN DER WERFF, H. \& F. LOREA. 1997. Lauraceae. En J. Rzedowski \& G. Calderón de Rzedowski (eds.), Flora del Bajío y de regiones adyacentes 56: 1-58. Instituto de Ecología, México.

VAN DER WERFF, H., C. A. ZANOTTI \& J. C. OSPINA. 2015. Lauraceae. En F. O. Zuloaga, M. J. Belgrano \& A. M. Anton (eds.), Flora Argentina 15: 41-57. Instituto de Botánica Darwinion, San Isidro.

VARADARAJAN, S., M. NARASIMHAN, M. MALAISAMY \& C. DURAIPANDIAN. 2015. In vitro anti-mycotic activity of hydro alcoholic extracts of some Indian medicinal plants against Fluconazole resistant Candida albicans. J. Clin. Diagn. Res. 9: ZC07-ZC10.

VIETZ, F. B. 1800. Icones plantarum medicooeconomico-technologicarum 1: tab. 61. Albrecht, Wien. 
G. Delucchi et al., Lauraceae naturalizadas en la Argentina

WILLIAMS, A. R., A. RAMSAY, T. V. HANSEN, H. M. ROPIAK, H. MEJER, P. NEJSUM, I. MUELLER-HARVEY, S. M. THAMSBORG. 2015. Anthelmintic activity of trans-cinnamaldehyde and A- and B-type proanthocyanidins derived from cinnamon (Cinnamomum verum). Sci. Rep. 5, doi: $10.1038 /$ srep 14791.

WILLIAMSON, J. 1967. Algunos árboles que se naturalizan en la Provincia de La Pampa, Argentina. Rev. Forestal Arg. 11: 45-50.
WU Z. \& HONG D. 2009. Flora of China Illustrations, vol. 7. Menispermaceae through Capparaceae. 520 pp. Science Press, Beijing-Missouri Botanical Garden Press, St. Louis.

YAP, P. S., T. KRISHNAN, K. G. CHAN \& S. H. LIM. 2015. Antibacterial mode of action of Cinnamomum verum bark essential oil, alone and in combination with piperacillin, against a multi-drug-resistant Escherichia coli strain. J. Microbiol. Biotechnol. 25: 1299-1306.

Original recibido el 13 de abril de 2016; aceptado el 10 de mayo de 2016. 
